\title{
Forbush-decrease in a Magnetic Cloud
}

\section{PETUKHOV, Ivan ${ }^{\mathbf{1}}$}

Yu.G. Shafer Institute of Cosmophysical Research and Aeronomy of SB RAS

31 Lenin ave., 677980 Yakutsk, Russia

E-maili_van@ikfia.sbras.ru

\section{PETUKHOV, Stanislav}

Yu.G. Shafer Institute of Cosmophysical Research and Aeronomy of SB RAS

31 Lenin ave., 677980 Yakutsk, Russia

E-mail petukhov@ikfia.sbras.ru

Calculation of cosmic ray intensity in a magnetic cloud is realized. It is supposed that the magnetic cloud near the Sun has the shape of a torus segment with typical structure of the magnetic field (magnetic field rope). The magnetic cloud is located in the coronal mass ejection having distribution of movement velocity by radius. The subsequent propagation of ejection in interplanetary space is determined on the basis of kinematic model. The magnetic field is determined by the freezing-in condition. It is supposed that the cosmic ray intensity in a magnetic cloud is determined by the large-scale electromagnetic field. The zero and first moments of distribution function of cosmic ray with different energies are calculated. It is revealed influence of the regions connecting a magnetic cloud to the Sun on cosmic ray intensity. Comparison of calculation results with measurements is shown.

The 34th International Cosmic Ray Conference

30 July- 6 August, 2015

The Hague, The Netherlands 


\section{Introduction}

It is generally believed that Interplanetary Coronal Mass Ejections (ICMEs) have the strongest influence on the condition of near-Earth space, geomagnetic activity and the intensity of Cosmic Rays (CRs). A Forbush decrease (FD) of CRs is caused by the stronger magnetic field in the disturbance region $[1,2]$. It was found that two-step FDs occur in ICMEs with a magnetic cloud (MC). Thus the second deeper decrease of intensity is due to the magnetic field rope (MFR) of a MC. The theoretical researches of FDs are of significance for the interpretation of measurement results. The diffusive models solving the transport equation for particles in diffusion approximation are used in the calculations. The $\mathrm{MC}$ is presented as a cylinder moving in interplanetary space with a constant radius in [3]. The expansion of the cylinder is considered in [4]. It is obtained that the FD model in which the MC has a form of the expansion cylinder in general correspondences with intensity measurements of CRs in some events [5]. The calculation results of FDs based on the model of the piston of a shock wave generated by a sharp speed jump of the solar wind are presented in [6]. The calculated FDs have the hard spectrum observed during the periods of solar minimum activity.

This work shows the FD kinetic model into the MC having the form of a torus segment with the MFR structure. The results demonstrate that the model reproduces the typical behavior of density and anisotropy of CRs in the MC.

\section{Model}

According to the kinematic approximation, the speed of Lagrangian particle flow persists that can be used for the calculation of a MC dynamic [7]. The approximation validity is based on: 1) the influence of the force-free magnetic field to the MC dynamic is negligible $(\vec{j} \times \vec{B} \ll 1) ; 2)$ the kinetic energy of the particle flow significantly exceeds the thermal energy $\left(M^{2}=u^{2} / c_{s}^{2}\right)$. Here $\vec{j}, \vec{B}$ are current and strength of the MC magnetic field, respectively; $M=u / c_{S}$ is the Much number; $u, c_{s}$ are the flow speed and the sound speed, respectively.

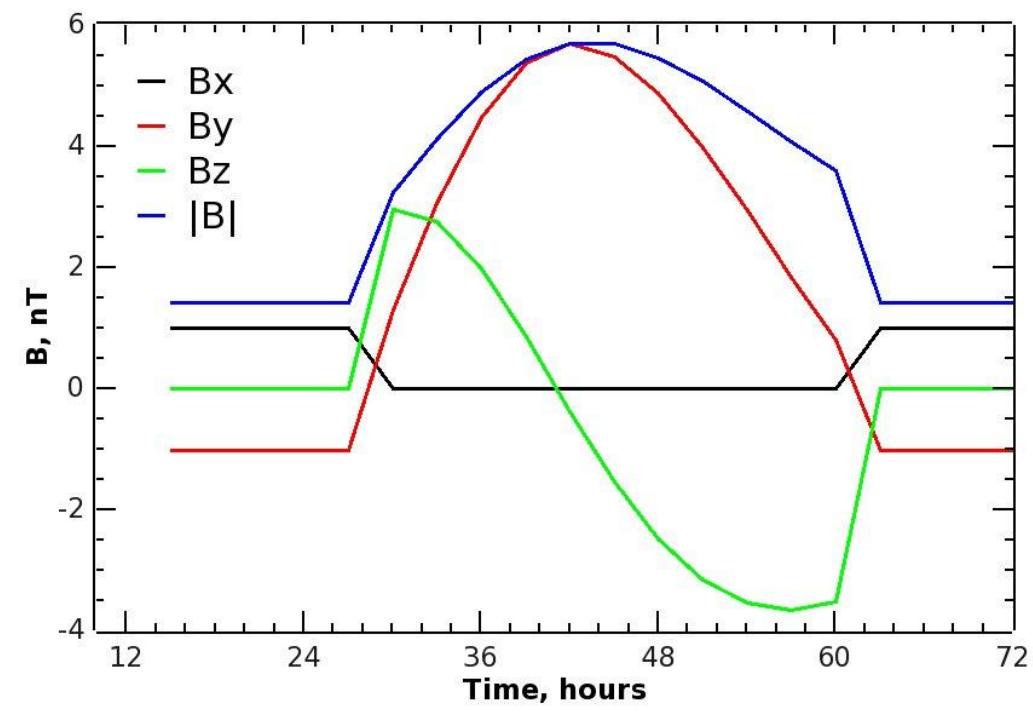

Figure 1. The magnetic field components and the module of moving MC at fixed point. 
It is suggested that the MC has the form of a torus segment at the initial time and its magnetic field corresponds to the solution [8]. The gradient of speed flow is specified. The change of the MC initial form calculated by the kinematic model. The magnetic field is determined by the freezing-in condition presented as the magnetic flow preservation through the area relating to the Lagrangian particle flow. In general, the model of the MC dynamic corresponds with the measurements as in the observed events the speed distribution of Lagrangian particles of the MC flow, as a rule, has a limited region. That is the reason of preservation of MFR structure.

The torus model used in the calculation includes next parameters: the section radius 0.16 $r_{e}$; the axis is located in the plane of the solar equator at $0.47 r_{e}$ from the Sun; the angular size is $\pi / 2$; the magnetic field components satisfy with the solution in [8]; the magnetic field strength on the torus axis is $20 \mathrm{nT}$; the MFR structure has the SWN type; the expansion of the torus by its movement is made by radial flow whose speeds of Lagrangian particles are distributed linearly by radius from $600 \mathrm{~km} / \mathrm{s}$ to $400 \mathrm{~km} / \mathrm{s}$. Parker wind surrounds the MC. The MC interaction with the solar wind isn't considered. Figure 1 illustrated the calculated components of the magnetic field of the MC model at fixed point located at $1 r_{e}$, where $r_{e}$ is the astronomical unit. It is confirmed the preservation of the MFR structure.

The particle distribution function without scatters remains along the particle trajectories. The trajectories are characteristics of the Boltzmann equation. Thus, the particle distribution function into the electromagnetic field is determined by calculation of a set of trajectories. Straight and back trajectories are used in the calculation [9]. The straight trajectories denote trajectories whose initial parameters are given at the source, i.e. where the distribution function is specified. The back trajectories end at the source respectively. The main distinction of the straight and the back trajectories consist in the sign of a time step for solution of the system of moving equations. The step is plus for the straight trajectories and it is minus for the back trajectories.

Real MCs are connected to the Sun by magnetic lines and the MC central part has the torus form. The significant part of the particles inside the MC comes to its feet due to the MFR structure. The influence of these regions is carried out by: 1) the torus segment is limited by ends; 2) the calculation of trajectories is performed only in the segment; 3) the full flow of particles coming to each end of the segment $I_{1}$ is calculated; 4) the flow of particles coming back from a end is $I_{2}=\alpha I_{1}$; where the values of reflection coefficient $\alpha=(1-0.98)$ are used; 5 ) it is suggested that the distribution function of the coming-back particles has an isotropic angular distribution and is homogenius over the end area. The flow properties of the comingback particles can be caused by the stronger magnetic field (reflection) and drift across the strong heterogeneous magnetic field of the MC feet (isotropy and homogeneity).

The calculation of distribution function in the torus segment is carried out in the following way. The flow of the particles coming from the environment to each of the ends is calculated by using of the back trajectories. By means of the given rules the flow of the coming-back particles is determined. Then the flow of particles passing from one end to another is calculated by the direct trajectories. During passing the particle flow decreases since a part of particles leaves to the environment. Thus, the recurrence relation between particle flows in successive passes between the ends is determined. The sum of flows defines a contribution of each ends. 
The source (the ends or the environment) is determined by the back trajectories for the calculation of the distribution function in any point located in the torus segment. The fraction of the relevant source is also found.

The distribution function $f(\vec{p})$ can be presented as $f(\vec{p})=f_{0}(p)+\vec{n} \overrightarrow{f_{1}}$ where $f_{0}, \overrightarrow{f_{1}}$ is isotropic and anisotropic parts of the distribution function, $\vec{n}$ is a single direction vector. The anisotropy expression is defined from the ratio $\vec{A}=-\overrightarrow{f_{1}} / f_{0}$. The density and anisotropy of CRs in the MC are obtained for the distribution function by several integrations: $f_{0}=\frac{1}{4 \pi} \int f d \Omega$; $f_{1 z}=\frac{3}{4 \pi} \int f \cos \theta d \Omega ; f_{1 x}=\frac{2}{\pi^{2}} \int f \cos \varphi d \Omega ; f_{1 y}=\frac{2}{\pi^{2}} \int f \sin \varphi d \Omega$, where $d \Omega$ is differential of solid angle. Angles $\theta, \varphi$ are counted off axis's Z and X, respectively.

\section{Results and Discussion}

Figure 2 shows the FD amplitude by time $A_{F D} . A_{F D}$ is calculated for the point in heliocentric coordinate system: $x=1 r_{e}, y=z=0$. The kinetic energy of particles is $\varepsilon_{k}=10 \mathrm{GeV}$, the magnetic field strength on the torus axis is $20 \mathrm{nT}$. The time step between any subsequent values is 3 hours. The lines correspond to the set of values $\alpha=1 ; 0.99 ; 0.98$. The chosen point is in the MC during 30 hours within 28th to 58th hours.

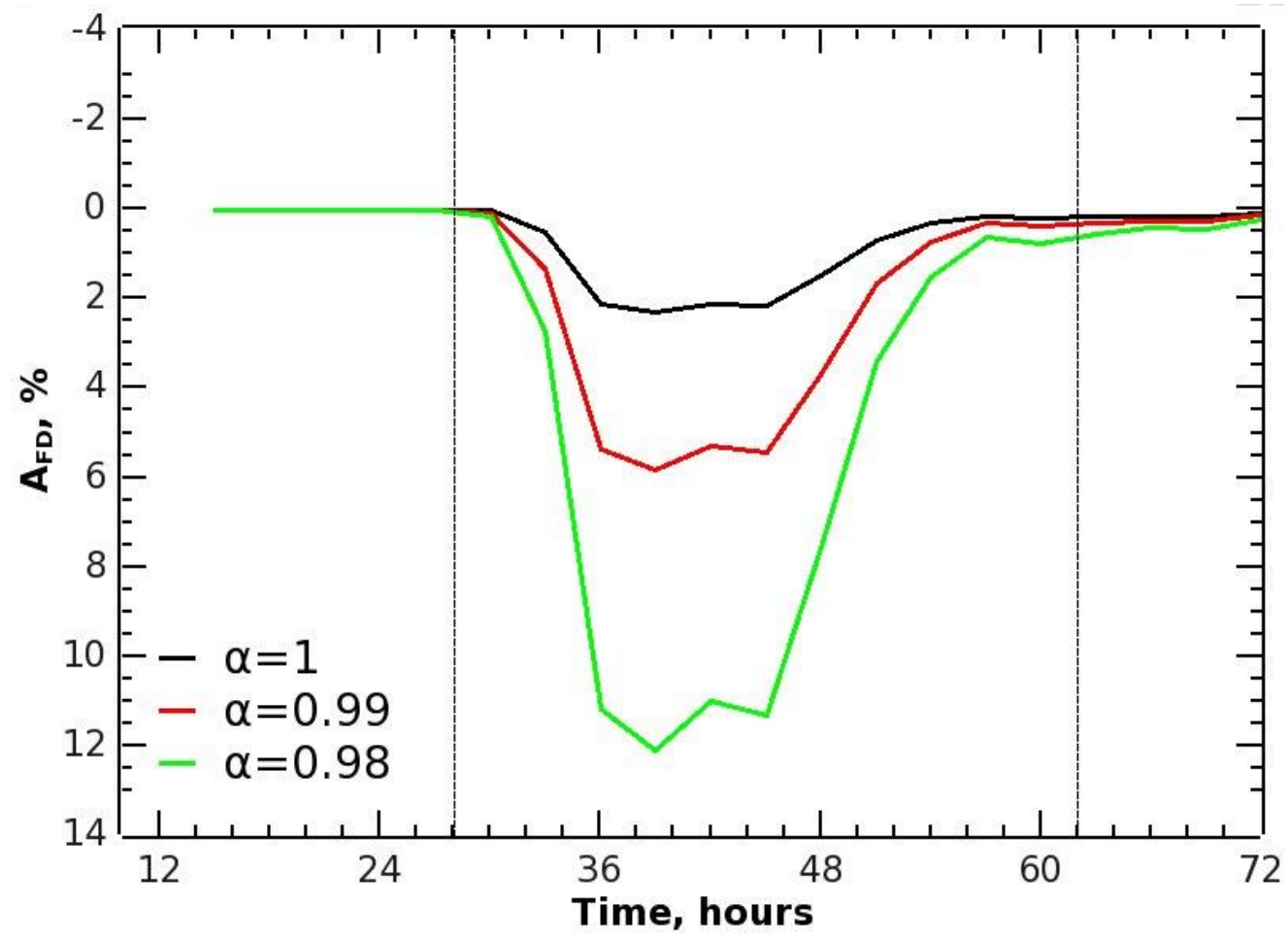

Figure 2. The FD amplitude by time for different values of reflection coefficient. Vertical black lines are MC boundaries. The particle kinetic energy is $10 \mathrm{GeV}$. The strength of the magnetic field on the torus axis is $20 \mathrm{nT}$.

As shown in the Figure, the decrease of FD is sharper than the recovery. The maximum value $A_{F D}$ changes from $2 \%$ to $12 \%$ thus, the more the particle losses at the segment ends, the more the value $A_{F D}$. Figures $3 \mathrm{a}, \mathrm{b}, \mathrm{c}, \mathrm{d}$ demonstrate the dependence of components and the 
module of the anisotropy by time. Figure 3a shows the anisotropy for $\alpha=1,3 \mathrm{~b}$ for $\alpha=0.99$, $3 \mathrm{c}$ for $\alpha=0.98$. Figure $3 \mathrm{~d}$ demonstrates the equatorial component of anisotropy $\left(A_{x}, A_{y}\right)$. The time behavior of anisotropy doesn't depend on the value $\alpha$.
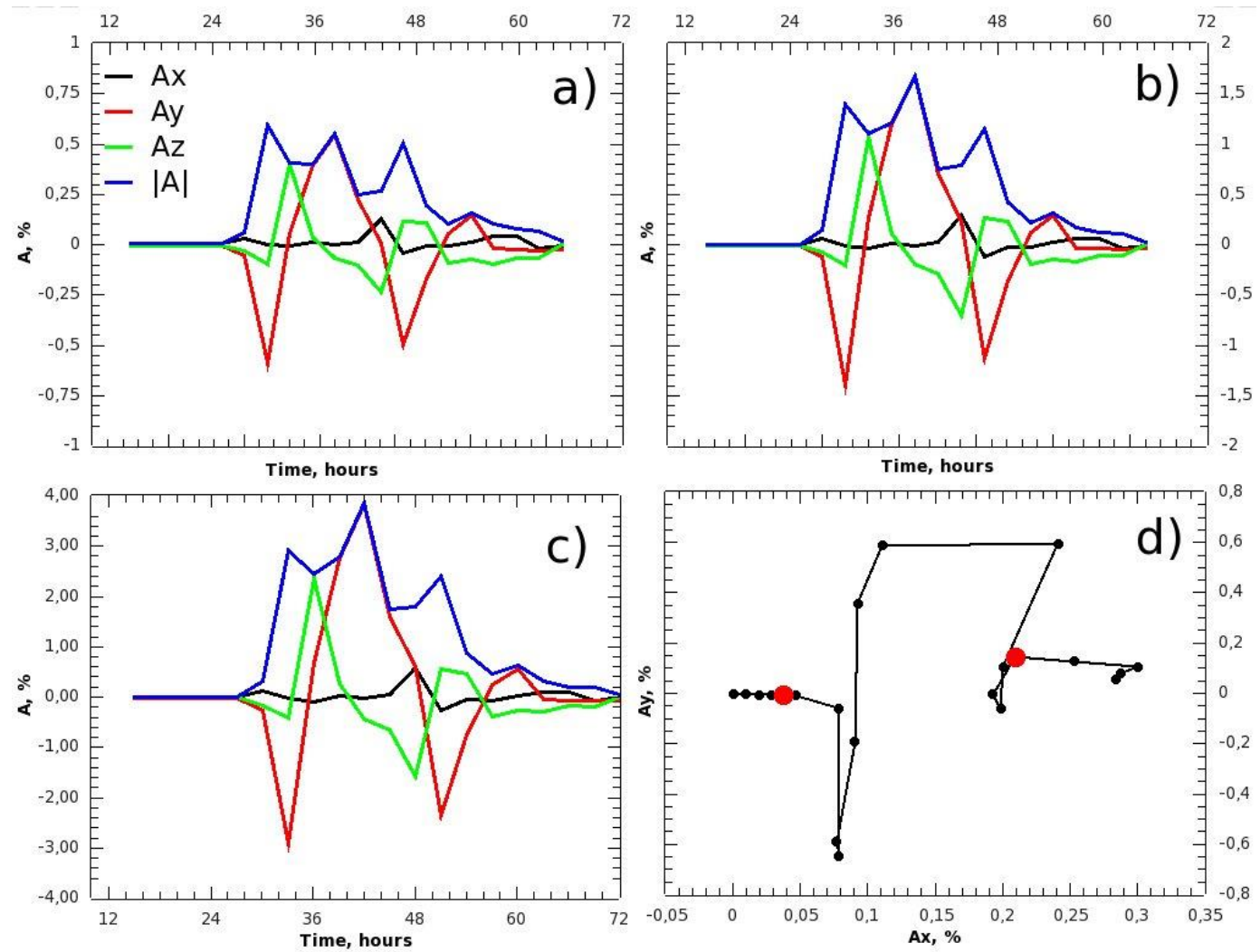

Figure 3. The components and module of anisotropy (a-c) and the equatorial component of anisotropy $\left(A_{x}, A_{y}\right)$ (d) by time. Red points are denote the entrance and exit from the MC.

However, the anisotropy amplitude depends on it: the less the value $\alpha$, the more the anisotropy. Each anisotropy component has a feature in behavior: $A_{x}$ is the smallest component, $A_{y}$ is the biggest component, it changes a sign two times passing through the MC; $A_{z}$ changes a sign once near the MC center. 


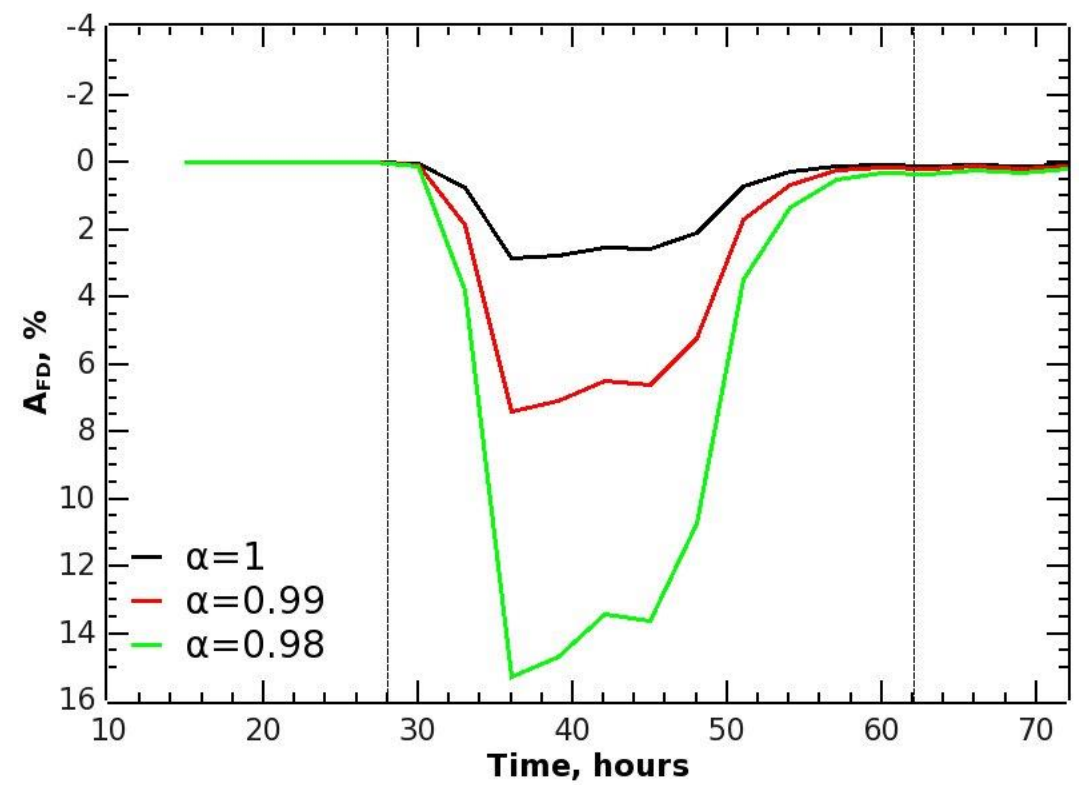

Figure 4. The FD amplitude by time. The particle energy is $10 \mathrm{GeV}$. The strength of the magnetic field on the torus axis is $40 \mathrm{nT}$.

The equatorial component of anisotropy shows rotation. There is an asymmetry of the anisotropy behavior: the time period from the beginning of change of the anisotropy (from entrance point of $\mathrm{MC}$ ) to the MC center is much shorter than the period from the center to its return to unperturbed value. Figure 4 shows the time dependence $A_{F D}$ for particles with $\varepsilon_{k}=10 \mathrm{GeV}$ and the value of the magnetic field on the torus axis $40 \mathrm{nT}$. Based on the comparison of Figure 2 and Figure 4 one can see that the increase of the magnetic field strength corresponds with the increase of the value $A_{F D}$. The behavior $A_{F D}$ for particles with $\varepsilon_{k}=$ $30 \mathrm{GeV}$ and the magnetic field $20 \mathrm{nT}$ is illustrated in Figure 5. According to the comparison in Figure 2, the value $A_{F D}$ decreases for particles with bigger energies.

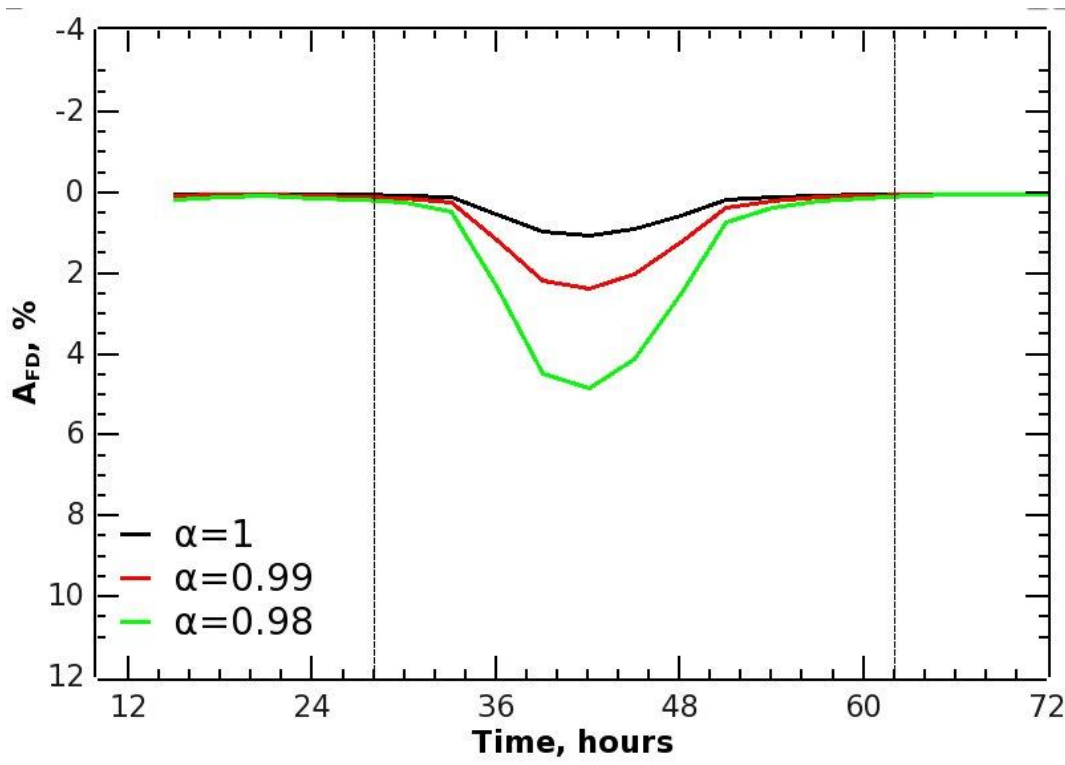

Figure 5. The FD amplitude by time. The particle energy is $30 \mathrm{GeV}$. The strength of the magnetic field on the torus axis is $20 \mathrm{nT}$. 
The results of the analysis of CR density and anisotropy with hardness $10 \mathrm{GV}\left(\varepsilon_{k}=\right.$ $9 \mathrm{GeV}$ ) in 99 events of the 23th and 24th solar cycles confirming the MCs presence are shown in [10]. In the studied sample, the MCs characteristics show a wide variety of the movement speed, the flow speed gradient in the MC, the distribution and the magnetic field value in the MC. The behavior of CR density and anisotropy in time are also variously. However, the typical behavior of density and anisotropy in the MC is determined: the value $A_{F D}$ is maximum in the MC; the decrease of density is sharp, the recovery is gradual; the anisotropy significantly changes at an entrance and/or an exit from the MC; the systematic change of anisotropy is often observed in the MC; rotation is typical for the equatorial component; the North-South component $\left(A_{z}\right)$ often changes a sign in the region of the MC center. Figures 2-5 show that the model calculations in general reproduce the behavior of the density and the anisotropy of CRs observed in MCs. However, the obtained characteristics of the FD doesn't correspond to the real FD in detail since it is considered that the MC is surrounded with the undisturbed environment in the calculation while, in fact, MCs are in ICME.

\section{Conclusion.}

1. The model for calculation the magnetic field in the MC located in a heterogeneous flow is suggested.

2. The calculation method of the distribution function in a MC using particle trajectories is developed.

3. The influence of the regions connecting a MC to the Sun on angular and spatial particle distribution in the $\mathrm{MC}$ is established. The approach taking into account the influence is suggested.

4. The time dynamics of density and anisotropy of the particle distribution function in the MC in general corresponding with measurements is calculated.

Acknowledgments

The work was supported by the Russian Foundation for Basic Research, grant no. 15-42-05085 r_vostok_a; and by the President of the Russian Federation, grant no. NSh-3269.2014.2.

\section{References}

[1] Parker E. Interplanetary Dynamical Processes. New York: Interscience Publisher, (1963). $272 \mathrm{p}$.

[2] Lockwood, J.A., Forbush Decreases in the Cosmic Radiation, Space Sci. Rev., 12, $658,1971$.

[3] Cane, H.V., I.G. Richardson and G. Wibberenz, The response of energetic particles to the presence of ejecta material, Proc. 24th Int. Cosmic Ray Conf. (Rome), 4, 377, (1995).

[4] Munakata K., et al., On the cross-field diffusion of galactic cosmic rays into an ICME, Adv. Geosci., 2, 115, (2006). 
[5] T. Kuwabara, J. W. Bieber, P. Evenson et al., Determination of Interplanetary Coronal Mass Ejection Geometry and Orientation from Ground-based Observations of Galactic Cosmic Rays, J. Geophys. Res., 114, A05109, (2009).

[6] F. Krymsky, P.A. Krivoshapkin, V.P. Mamrukova and S.K. Gerasimova. North-south asymmetry of the heliosphere from cosmic-ray observations. // Astron. Lett. 35. 5. P.333337. 2009.

[7] I. S. Petukhov and S. I. Petukhov, Transparency of a Magnetic Cloud Boundary for Cosmic Rays, Bull. Russ. Acad. Physics, 77, 5, pp. 523-525 (2013).

[8] Miller G., Turner L., Force free equilibria in toroidal geometry, Phys. Fluids, 24, 363 (1981).

[9] Petukhov I.S., S.I. Petukhov, Galactic Cosmic Ray Intensity Dynamics in the Presence of Large-Scale Solar Wind Disturbances, Astronomy Letters, 35, 10, pp. 701-711 (2009).

[10] Abunin A.A., Abunina M.A., Belov A.V., Eroshenko E.A., Oleneva V.A., Yanke V.G., Papaioannou A., Mavromichalaki H., Impact of magnetic clouds on the density and the first harmonic of cosmic ray anisotropy, Solar physics and near space. Proceedings of the AllRussian Conference on Solar-Terrestrial Physics, dedicated to the 100th anniversary of the birth of V.E.Stepanov. Irkutsk. P. 11-14. (2013). (In russian). 